\title{
Microbial and enzyme technology: An efficient and convenient method for MiniPrep analysis of recombinant plasmids
}

\author{
Masahiro Sato $^{1}$, Emi Inada $^{2}$, Issei Saitoh ${ }^{3}$, Yuko Matsumoto ${ }^{2}$ \\ ${ }^{1}$ Section of Gene Expression Regulation, Frontier Science Research Center, Kagoshima University, Sakuragaoka, Kagoshima, Japan \\ ${ }^{2}$ Department of Pediatric Dentistry, Kagoshima University Graduate School of Medical and Dental Sciences, Sakuragaoka, Kago- \\ shima, Japan \\ ${ }^{3}$ Division of Pediatric Dentistry, Graduate School of Medical and Dental Science, Niigata University, Gakkocho-dori, Chuo-ku, Nii- \\ gata, Japan \\ Email: masasato@ms.kagoshima-u.ac.jp, inada@dent.kagoshima-u.ac.jp, isaito@dent.niigata-u.ac.jp, mat@dent.kagoshima-u.ac.jp
}

Received 25 December 2013; revised 31 January 2014; accepted 7 February 2014

Copyright (C) 2014 Masahiro Sato et al. This is an open access article distributed under the Creative Commons Attribution License, which permits unrestricted use, distribution, and reproduction in any medium, provided the original work is properly cited. In accordance of the Creative Commons Attribution License all Copyrights (c) 2014 are reserved for SCIRP and the owner of the intellectual property Masahiro Sato et al. All Copyright (C) 2014 are guarded by law and by SCIRP as a guardian.

\section{ABSTRACT}

Minipreparation (MiniPrep) analysis is an essential step for obtaining a recombinant plasmid that carries a DNA insert containing a gene of interest. The most commonly used method for this involves cultivation of transformed Escherichia coli $(E$. coli) in liquid medium, brief centrifugation for precipitation of bacterial pellets, and subsequent lysis of the pellets. This process is time-consuming and laborious, especially when the sample number is high. Here, we describe a more convenient method for MiniPrep analysis that utilizes solid medium-based cultivation of bacteria.

\section{KEYWORDS}

Agar Plate; E. coli; MiniPrep Analysis; Gene Engineering; Plasmid

\section{INTRODUCTION}

Gene cloning is widely used as an important tool for obtaining a gene-engineered plasmid carrying an insert with a gene of interest [1]. It involves cultivation of transformed Escherichia coli (E. coli), collection of bacterial pellets, and lysis of these pellets for MiniPrep analysis.

Cultivation of E. coli cells in liquid medium containing antibiotics is one of the most commonly employed methods for isolation of large amounts of plasmids [1-3]. Specifically, cultivation in $1-2 \mathrm{ml}$ of the liquid medium with gentle shaking for plasmid isolation is often performed. However, this method requires that E. coli cells be precipitated by centrifugation with an ultra-rapid centrifuge, which is generally used for the precipitation of plasmid DNA in 1.5 - 2-ml microcentrifuge tubes. However, this process is time-consuming and laborious, especially when a large number of samples have to be treated.

Here, we describe a more convenient method for MiniPrep analysis that utilizes solid medium-based cultivation of bacteria, as previously suggested by ours [4]. This method does not require collection of bacterial pellets by centrifugation of liquid suspensions; instead, it requires toothpick-based transfer of bacterial clots grown on agar plates to a 1.5-ml tube. These collected samples are then subjected to lysis to recover plasmids from $E$. coli cells. For enzymatic analysis of these MiniPrep-derived plasmids, the crude lysates were subjected to further purification using column-based DNA purification kits $[2,3]$.

However, this process can become time-consuming with a larger number of samples. Therefore, we recommend pre-screening of recombinants through $0.8 \%$ agarose gel electrophoresis of intact crude extracts after bacterial lysis and subsequent phenol-based clearance of proteins. This allows us to distinguish recombinants from non-recombinants based on the difference in their molecular weights, since non-recombinants migrate faster than recombinants. Thus, we selected recombinants for further analysis to confirm whether they have proper inserts through restriction enzyme analysis.

\section{METHODS}

To verify the above-mentioned method for MiniPrep DNA analysis, an example is shown below. First, the 
PCR-amplified 320-bp insert was cloned into a TA cloning vector pCR2.1 (Invitrogen Co., Carlsbad, CA). Briefly, $2 \mu$ l of PCR fragments ( 20 ng) was mixed with $1 \mu \mathrm{l}$ of pCR2.1 and $3 \mu$ l of Ligation High (Toyobo, Tokyo, Japan) that contained DNA ligase, and the mixture was left at $16^{\circ} \mathrm{C}$ for over $30 \mathrm{~min}$. For transformation of $E$. coli, $1 \mu \mathrm{l}$ of the ligation mixture was mixed with $7 \mu$ of $E$. coli suspension. DH5 $\alpha$ competent cells (Takara Bio Inc., Shiga, Japan). The mixture was placed for 20 min on ice/ water, heat-activated for $1 \mathrm{~min}$ at $42^{\circ} \mathrm{C}$, and $70 \mu \mathrm{l}$ of sterile Plusgrow (Nacalai-Tesque Inc., Kyoto, Japan) was added to recover $E$. coli through incubation for $20 \mathrm{~min}$ at $37^{\circ} \mathrm{C}$.

Then, the bacteria were spread onto the corner of $2 \%$ agar plate [containing 2\% solid agar (Nacalai-Tesque Inc.), and Plusgrow] in a $100-\mathrm{mm}$ bacteriological petri dish (Eiken Kizai Co., Tokyo, Japan) that had been coated with $10 \mathrm{mg}$ of ampicillin (Invitrogen Co.) $\mathrm{ml}^{-1}$ and 4 mg of X-Gal (5-bromo-4-chloro-3-indolyl- $\beta$-D-galactoside; Invitrogen Co.) $\mathrm{ml}^{-1}$. The plate was incubated overnight at $37^{\circ} \mathrm{C}$ to obtain bacterial colonies. Several white or blue colonies were picked up by sterile toothpicks and spread onto the surface of the plate, next to other transformed colonies (shown by the red quadrants in the upper panel of Figure 1(a)).

One day after spreading, about one-half of the resulting colonies (enclosed by red quadrants in the middle panel of Figure 1(a)) were picked up and transferred to a 1.5-ml tube. The collected cells were then lysed to release the plasmid by dissolution in $20 \mu \mathrm{l}$ of the lysis buffer STET (comprising 8\% sucrose, 5\% Triton X-100, 50 mM EDTA, and $50 \mathrm{mM}$ Tris, pH 8.0). Samples were then vortexed and the lysed debris was then mixed with $3 \mu \mathrm{l}$ of saturated phenol for extraction. After vortexing, these mixtures were separated from the plasmid-containing supernatant by centrifugation with an ultra-rapid centrifuge (MX-100; Tomy Seiko Ltd., Tokyo, Japan) for 2 min at $16,000 \times \mathrm{g}$ at $4^{\circ} \mathrm{C}$. Approximately $10 \mu \mathrm{l}$ of the collected supernatant was then subjected to $0.8 \%$ agarose gel electrophoresis with pCR2.1 DNA ( 300 ng) as the negative control.

The remaining bacterial clots from the selected colonies were then picked up by toothpicks (as shown in the white quadrant in the lower panel of Figure 1(a)) and subjected to further purification using the MACHEREYNAGEL MiniPrep kit (Takara Bio Inc.). The DNA attached to the column was eluted by $30 \mu$ l of buffer EB. Two microliters of the purified DNA was digested with Eco RI (Toyobo, Tokyo, Japan) to release the 320-bp insert prior to $2 \%$ agarose gel electrophoresis.

\section{RESULTS AND DISCUSSION}

Among the four isolated bacterial colonies, two (lanes 2 and 3 in Figure 1(b)) were identified as recombinants, (a)

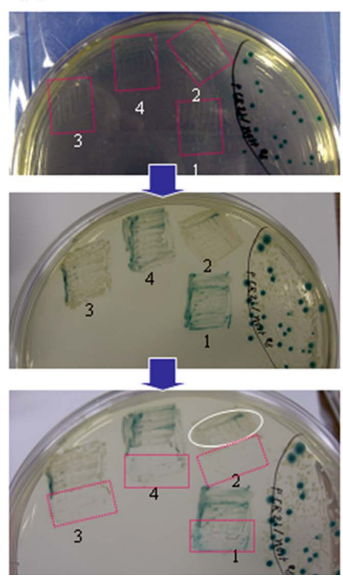

(b) Intact MiniPrep DNA samples

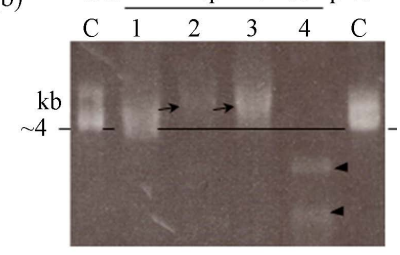

(c)

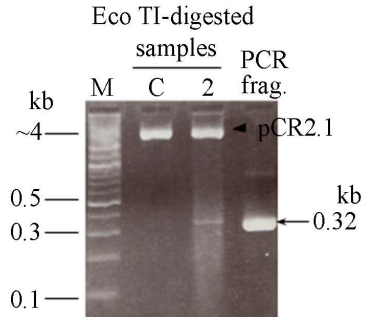

Figure 1. A simplified MiniPrep analysis. (a) Procedure for MiniPrep analysis from solid medium-based plasmid recovery. (b) Gel electrophoresis of crude plasmid DNA extracts from an agar plate culture. Arrows indicate colonies carrying inserts. Arrowheads indicate ribosomal RNAs. (c) control plasmid (pCR2.1). (c) Restriction enzyme analysis of Eco RI-digested column-purified plasmid DNA recovered from an agar plate culture. M, 100-bp ladder markers. C, control plasmid (pCR2.1). 2, plasmid DNA from the colony in lane 2 of (b). PCR frag., 320-bp PCR-amplified fragment used for gene cloning. Note the presence of plasmid backbones of $\sim 4 \mathrm{~kb}$ in lanes $\mathrm{C}$ and 2 after digestion with Eco RI.

since they migrated slower than the parental pCR2.1 vector (arrows in lanes 2 and 3 vs. lane $C$ in Figure 1(b)). The colony in lane 1 appears to be non-recombinant, since it migrated at the same speed as the control. The colony in lane 4 appears to have lost plasmids during cultivation: only two ribosomal components (arrowheads in Figure 1(b)) are visible. We thus decided to purify the colony in lanes 2 and 3 for further enzymatic analysis. Digestion with Eco RI of the purified DNA revealed that the colony in lane 2 had an insert whose size is equivalent to that of the PCR-amplified insert (lane 2 vs. PCR frag. in Figure 1(c)). The DNA in lane 3 had also a 0.32$\mathrm{kb}$ insert (data not shown). As expected, the control pCR2.1 plasmid (lane C in Figure 1(c)) did not release any fragments.

Previous protocols [5,6] necessitate that MiniPrep starts with 1 - $5 \mathrm{ml}$ of an overnight bacterial culture in a liquid medium. Consequently, it always requires centrifugation to precipitate bacterial clots prior to lysis of bacteria, and this step is labor-intensive and time-consuming. The present procedure does not require that type of bacterial collection. Instead, bacterial clots can be picked up easily by sterile toothpicks.

Another merit of this improved method is pre-screening of candidate recombinants by loading crude DNA extracts immediately after lysis in $0.8 \%$ agarose gels. This electrophoresis makes it possible to identify recombinants after ethidium bromide staining of gels. This prescreen- 
ing procedure would be valuable for cloning/transfection experiments with limited financial resources.

\section{CONCLUSION}

Agar-based cultivation of bacteria is a more convenient method for MiniPrep analysis. Furthermore, pre-screening of recombinants by electrophoresis in $0.8 \%$ agarose gels as crude DNA extracts is proven useful for selection of recombinants carrying a target insert.

\section{ACKNOWLEDGEMENTS}

This work was supported by a grant-in-aid from the Ministry of Education, Science, Sports, Culture, and Technology of Japan.

\section{REFERENCES}

[1] Sambrook, J., Fritsch, E.F. and Maniatis, T. (1989) Molecular cloning: A laboratory manual. 2nd Edition, Cold
Spring Harbor Laboratory Press, Cold Spring Harbor.

[2] DNA Purification in Promega Home Page. (http://www.promega.com/resources/product-guides-andselectors/protocols-and-applications-guide/dna-purification/

[3] QIAGEN Plasmid Purification System in Qiagen Home Page.

http://www.ebiotrade.com/buyf/productsf/qiagen/QIAGE N plasmid purification system.htm

[4] Sato, M., Akasaka, E., Saitoh, I., Ohtsuka, M., Nakamura, S., Sakurai, T. and Watanabe, S. (2012) A simplified protocol for the semi-large scale recovery of plasmids from Escherichia coli grown on agar plates. Journal of Biomedical Science and Engineering, 5, 406-408. http://dx.doi.org/10.4236/jbise.2012.57051

[5] DNA Plasmid Miniprep Protocol. http://www.protocol-online.org/cgi-bin/prot/view_cache.c gi?ID=3062

[6] Mini-prep Protocol. http://irc.igd.cornell.edu/Protocols/Mini-prep.htm 